\title{
Transformer Fault Position Recognition Based on Probability Support Vector Machine
}

\author{
Huang Song-bo ${ }^{1, a}$, Zhao Wei-min ${ }^{1, b}$, Zhang Tao ${ }^{1, c}$, Sima Li-ping ${ }^{2, d}$, Wang Bo ${ }^{2, e}$ \\ and Shu Nai-qiü, \\ ${ }^{1}$ Foshan Power Supply Bureau, Guangdong Power Grid Corporation, Foshan, China \\ ${ }^{2}$ School of Electrical Engineering, Wuhan University, Wuhan, China \\ a13702933073@139.com, 'Winner-1@163.com, 'Tonymemo@126.com, ${ }^{\mathrm{d}}$ smlping@163.com, \\ ewhwdwb@gmail.com, 'shunaiqiu1@163.com
}

Keywords: transformer; fault recognition; fault position; SVM; posterior probability

\begin{abstract}
Based on posterior probability of support vector machine, the analysis of dissolved gases in oil and the data of electrical tests are used comprehensively to recognize internal fault positions of the power transformer. This method not only inherits the advantages of the support vector machine for small samples, strong generalization ability and so on, but also provides the fault information of the power transformer by the form of probability. The output results not only provide the breaking down probability of the inner winding, tap changer and leads, iron core, structure and magnetic shielding body, insulating barrier and other parts, but also express the degree of credibility of conclusions. It adapts to the uncertainty characteristics of the fault positions. After analysis of examples, the validity of the model is verified.
\end{abstract}

\section{Introduction}

In recent years, fault diagnosis for transformer has become one of the research hotspots in electric power industry. At present, support vector machine theory based on the structural risk minimization principle has some achievements ${ }^{[1-3]}$ in the matter of dealing with the traditional transformer fault intelligent diagnosis methods of small sample, nonlinear, local minima and so on. Standard SVM results are hard-decision output and provide clear category information, but the transformer fault is a dynamic development process, the fuzzy diversity of fault reasons, phenomena and mechanism and the structure complexity of transformer make the diagnosis results performed in an uncertain form, which is closer to the real situation of the fault. On the other hand, the current diagnostic contents focus on the property and degree of discharge or overheating fault diagnosis, lacking of diagnosis of the fault positions, which are more meaningful to the transformer.

Aiming at these problems, a probability estimation model for power transformer internal fault is put forward in the paper. This model based on the SVM posterior probability theory makes a probability estimation of transformer internal positions and gives diagnosis conclusions in probability form.

\section{Theory of Posterior Probability Support Vector Machine}

Support vector machine is a machine learning method that based on statistical learning theory, which has a strict mathematical foundation, because it depends on the structural risk minimization principle, so the support vector machine classifier has a better generalization ability and has been widely practical applied ${ }^{[4]}$.For the linearly separable training set, supposing that there exist the training samples $(x 1, y 1), \ldots,(x 1, y 1), x \in R^{n}, y \in\{+1,-1\}, l$ is the number of samples, $n$ is the input dimension. On the linearly separable condition, there are a hyperplane that makes two kinds of samples completely separated, the hyperplane is described as

$$
w \cdot x+b=0
$$


Finding the optimal hyperplane can be viewed as the quadratic planning problems. For given training samples, finding the optimal value of the weight $W$ and offset $b$ can make the weighted cost function minimize, namely:

$$
\left\{\begin{array}{l}
\min \frac{1}{2}\|w\|^{2} \\
y_{i}\left(w \cdot x_{i}+b\right) \geq 1, \quad i=1,2, \ldots, l
\end{array}\right.
$$

When the training sample set can not be linearly separated, non-negative slack variables are introduced. The optimization problem of separating hyperplane can be described as:

$$
\left\{\begin{array}{l}
\min \frac{1}{2}\|w\|^{2}+C \sum_{i=1}^{l} \xi_{i} \\
y_{i}\left(w \cdot x_{i}+b\right) \geq 1-\xi_{i}, \quad \xi_{i} \geq 0, i=1,2, \ldots, l
\end{array}\right.
$$

$C$ is the penalty parameter, the greater $C$, the greater the penalty for misclassification.

By solving the optimization problem, we can get the optimal decision function

$$
f(x)=\operatorname{sgn}\left[\sum y_{i} a_{i}<x \cdot x_{i}>+b\right]
$$

For a given input, the samples provided by standârd SVM whether belongs to a category or not,that is, a category's two extreme cases of the probability is 1 or 0 ,and do not provide the posterior probabilities output.Posterior probability is a very important index, Duda and Hart pointed out that in pattern recognition, the posterior probability, to a certain extent, improve the performance of the classifier. Wahba and Platt first introduced the posterior probability to the standard support vector machine method ${ }^{[5]}$.Through the conclusion and the empirical data analysis, using the sigmoid function parameter model that can directly fit posterior probability, Platt maps the standard SVM output value $\mathrm{f}$ into a probability value $p(y=1 \mid f)$, that is

$$
p(y=1 \mid f)=\frac{1}{1+e^{A f+B}}
$$

In the formula, according to the training set, parameter $A, B$ can be found through settling a maximum- likelihood problem:

$$
F(A, B)=\min \left(-\sum_{i=1}^{l} t_{i} \log \left(p_{i}\right)+\left(1-t_{i}\right) \log \left(1-p_{i}\right)\right)
$$

\section{Transformer Fault Position Recognition Based on Posterior Probability SVM}

\section{A. Feature Extraction And Data Preprocessing}

Different characteristics of signals reflect the real situation of transformer fault from different aspects. According to collected fault data and studying reference [6], fault features are chromatographic characteristic, ratio of $\mathrm{CO} / \mathrm{CO}_{2}$, core insulation, three-phase unbalance rate of winding DC resistance, winding ratio deviation, winding absorption ratio or polarization index, water content in oil. Considering the difference and dispersion of all the test data, in order to avoid the variable quantity level difference is so large that impacts results, the original data are" fuzzy", the fuzzy membership functions are ascending/descending trapezoid distribution function.

\section{B. Fault Position Classification}

Transformer internal faults can be divided into three main categories ${ }^{[7]}$ of circuit fault, magnetic circuit fault and abnormalities in oil. Each kind of fault can be subdivided. This paper combined with the internal structure of transformer determines the fault types as shown in Table 1.

Table 1. Fault position classification

\begin{tabular}{|l|l|l|}
\hline Fault Loop & Code & Fault Position \\
\hline \multirow{2}{*}{ Circuit } & P1 & Winding \\
\cline { 2 - 3 } & P2 & Tap Changer and leads \\
\hline \multirow{3}{*}{ Magnetic Circuit } & P3 & Iron core \\
\cline { 2 - 3 } & P4 & Structure and magnetic shielding body \\
\hline \multirow{3}{*}{ Abnomalities in Oil } & P5 & Insulating barrier and stay \\
\cline { 2 - 3 } & P6 & Floating potential body \\
\cline { 2 - 3 } & P7 & Others \\
\hline
\end{tabular}




\section{PPSVM-based Transformer Fault Position Identification Steps}

Power transformer internal fault position recognition based on the PPSVM is divided into two stages: training stage and diagnosis stage. The main steps are as follows: 1)Obtaining training samples, then fuzzy pretreatment; 2) According to the seven fault types , using the two-two paired to form 21 two SVM classifications, to obtain the optimal parameters by genetic algorithm, and using the best parameter training, to obtain 21 two SVM classification probability estimation models; 3) For the test sample,using 21 SVM probability estimation models to calculate probability; 4)Collecting the 21 SVM two-two paired posterior probability, solve the optimal problems to obtain each probability estimate.

\section{SVM Kernel Function And Optimal Parameters}

The choice of the kernel function and parameter setting are the key of SVM training. This paper selects the Gaussian radial basis function $K\left(x, x_{i}\right)=\exp \left(-g\left\|x-x_{i}\right\|^{2}\right)$. The parameters needed to determine are: penalty coefficient $C$, relaxation factor $\mathcal{E}$ and kernel function parameter $g$. This article take $\varepsilon=10^{-4}$, obtains parameter $C$ and $g$ by using genetic algorithm.

Genetic algorithm applies the nature biological evolution principle of "survival of the fittest, survival of the fittest" to optimize the encoding parameters in series to form groups, according to the selected fitness function and through genetic selection, crossover and mutation screening of the individual, makes the fitness values of good individual reserved, the individual of poor fitness eliminated, the new group not only inherited the past generation information, but better than the last generation. This paper takes the training under cross-validation fault recognition rate as the fitness function. Select the having highest recognition rate $C$ and $g$ as the training function of SVM model. Figure 1 shows the results that optimized under the 142 training samples. It can be seen from the figure, the optimal parameter $C=5.64, g=0.23$, the highest cross-validation accuracy is $91.39 \%$.

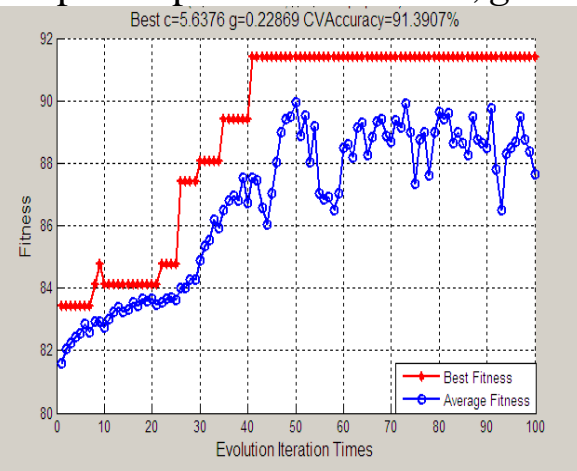

Fig. 1 Ggenetic algorithm performance tracking chart

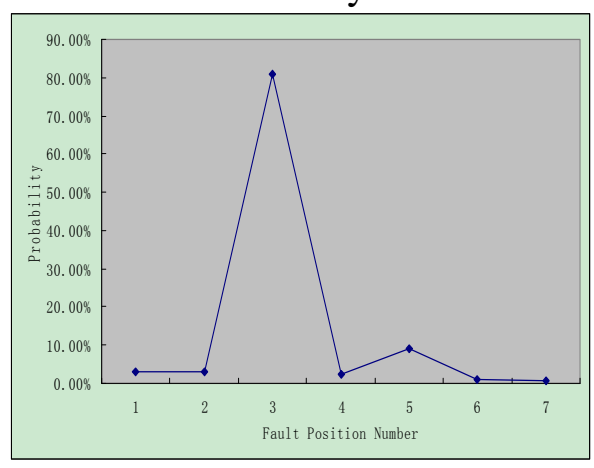

Fig. 2 Probability distribution of fault positions

\section{Case Studies}

\section{A. Recognition Results Analysis}

213 groups transformer fault data with clear conclusions is collected from power supply bureau and the literatures.142 groups are selected as the training samples, the other 71 groups as the test samples. Table 2 shows diagnosis results with maximum a posteriori probability principle.

Table 2. Fault samples distribution and recognition results

\begin{tabular}{|c|c|c|c|c|c|c|c|}
\hline Fault Position & P1 & P2 & P3 & P4 & P5 & P6 & P7 \\
\hline Total number & 51 & 29 & 53 & 35 & 18 & 8 & 8 \\
\hline Training number & 36 & 20 & 38 & 25 & 13 & 5 & 5 \\
\hline Test number & 18 & 10 & 19 & 12 & 6 & 3 & 3 \\
\hline right decision number & 15 & 9 & 17 & 12 & 5 & 2 & 3 \\
\hline Recognition rate(\%) & 83.3 & 90 & 89.5 & 100 & 83.3 & 66.7 & 100 \\
\hline Total recognition rate & \multicolumn{7}{|c|}{$88.73 \%(63 / 71)$} \\
\hline
\end{tabular}

Data in Table 2 show that the model has good generalization. Diagnosis results fit actual results well. Total recognition rate is up to $88.73 \%$. Especially for the circuit and magnetic circuit fault it has a good recognition rate.

\section{B. Fault Recognition Case}


The chromatographic analysis of a 500kV transformer in Foshan substation in June 2001 shows that contents of total hydrocarbon and acetylene are abnormal. Data is shown in Table 3. Core grounding current is $0.25 \mathrm{~A}$, and other tests are normal.

\begin{tabular}{|c|c|c|c|c|c|c|}
\hline $\mathrm{H}_{2}$ & $\mathrm{CH}_{4}$ & $\mathrm{C}_{2} \mathrm{H}_{6}$ & $\mathrm{C}_{2} \mathrm{H}_{4}$ & $\mathrm{C}_{2} \mathrm{H}_{2}$ & $\mathrm{CO}$ & $\mathrm{CO}_{2}$ \\
\hline 73.9 & 107.6 & 26.3 & 148.3 & 1.3 & 256.6 & 912.6 \\
\hline
\end{tabular}

Fault probability of each part is calculated by SVM probability estimation model. Diagnosis results are shown in Table 4. Actual Fault is that the yoke shield fastening bolt next to core broken, which causes the shielding plate falling off, forms multiple grounding, and discharges intermittently. Fault diagnosis result is reconciled with the actual fault. Probability distribution is also shown in Figure 2. It can be seen that SVM-based probability estimation model not only has a better recognition rate, but also has a good probability distribution.

Table 4. Diagnosis results

\begin{tabular}{|c|c|c|c|c|c|c|c|}
\hline P1 & P2 & P3 & P4 & P5 & P6 & P7 & Diagnosis Results \\
\hline 0.03 & 0.03 & 0.81 & 0.02 & 0.09 & 0.01 & 0.01 & Core fault \\
\hline
\end{tabular}

\section{Conclusions}

A SVM-based transformer internal fault position probability estimation model is proposed in this paper. The model has the following characteristics:1) Compared with fault diagnosis based on single feature, it makes full use of the complementary information of dissolved gas in oil and electrical tests data.2)SVM diagnosis results with probability information overcome the defects of hard-decision output and present the diagnosis results in quantify form, it not only improves the fault recognition rate, but also have a good probability distribution3)The diagnosis results in the form of probability not only give the probability of each fault position, but also express the credibility of diagnosis conclusion, and adapt to the transformer fault uncertainty characteristics.

\section{References}

[1] GUO Chuang-xin,ZHU Cheng-zhi,ZHANG Lin,etc. A Fault Diagnosis Method for Power Transformer Based on Multiclass Multiple-kernel Learning Support Vector Machine[J]. Proceedings of the CSEE. 2010, 30(13): 128-134.

[2] LIU Tong-jie,LIU Zhi-gang,HAN Zhi-wei,etc. Application of adaptive fuzzy support vector machine with ajacent incremental algorithm of transformer fault diagnosis[J]. Power System Protection and Control. 2010, 38(17): 47-52.

[3] Liping SIMA, Naiqiu SHU. Fault Diagnosis of Power Transformer Based on Clustering Binary Tree SVMs [C]. 2011 IEEE International Conference on Electric Information and Control Engineering, Wuhan, China. 2011, ICEICE 2011, pp: 5035-5038 .

[4] Vapnik. V N. The Nature of Statistical Learning Theory[M]. New York: Springer-Verlag, 1995.

[5] Platt J C. Probabilistic Outputs for Support Vector Machines and Comparison to Regularized Likelihood Methods.[M]. Advances in Large Margin Classifiers, A. Smola P B B S, Cambridge, MA, 2000. MIT Press.

[6] YANG Li,SHANG Yong,ZHOU Yue-feng,etc. Probability Reasoning and Fuaay Techique Applied for Identifying Power Transformer Malfunction[J]. Proceedings of the CSEE. 2000, 20(7): 20-24.

[7] WEI Xin,SHU Nai-qiu,CUI Peng-chen,etc. Power Transformer Fault Integrated Diagnosis Based on Improved PSO-BP Neural Networks and D-S Evidential Reasoning[J]. Automation of Electric Power Systems. 2006, 30(7): 46-50. 